\title{
Chchd2 regulates mitochondrial morphology by modulating the levels of Opa1
}

\author{
Wei Liu ${ }^{1,2} \cdot$ Xiuying Duan ${ }^{1,2} \cdot$ Lingna $\mathrm{Xu}^{1,2} \cdot$ Weina Shang ${ }^{2} \cdot$ Jiayao Zhao ${ }^{1} \cdot$ Liquan Wang $^{1} \cdot$ Jian-Chiuan $\mathrm{Li}^{4}$. \\ Chun-Hong Chen ${ }^{4} \cdot$ Jun-Ping Liu ${ }^{3} \cdot$ Chao Tong ${ }^{1,2,3}$
}

Received: 14 April 2019 / Revised: 10 December 2019 / Accepted: 10 December 2019 / Published online: 6 January 2020

(c) The Author(s), under exclusive licence to ADMC Associazione Differenziamento e Morte Cellulare 2020

\begin{abstract}
The mitochondrion is a highly dynamic organelle that is critical for energy production and numerous metabolic processes. Drosophila Chchd2, a homolog of the human disease-related genes CHCHD2 and CHCHD10, encodes a mitochondrial protein. In this study, we found that loss of Chchd2 in flies resulted in progressive degeneration of photoreceptor cells and reduced muscle integrity. In the flight muscles of adult Chchd2 mutants, some mitochondria exhibited curling cristae and a reduced number of cristae compared to those of controls. Overexpression of Chchd 2 carrying human disease-related point mutations failed to fully rescue the mitochondrial defects in Chchd2 mutants. In fat body cells, loss of Chchd2 resulted in fragmented mitochondria that could be partially rescued by Marf overexpression and enhanced by Opa1 RNAi. The expression level of Opa1 was reduced in Chchd2 mutants and increased when Chchd2 was overexpressed. The chaperonelike protein P32 co-immunoprecipitated with Chchd2 and YME1L, a protease known to processes human OPA1. Moreover, the interaction between P32 and YME1L enhanced YME1L activity and promoted Opa1 degradation. Finally, Chchd 2 stabilized Opa1 by competing with P32 for YME1L binding. We propose a model whereby Chchd2 regulates mitochondrial morphology and tissue homeostasis by fine-tuning the levels of OPA1.
\end{abstract}

\section{Introduction}

Mitochondria are the central hubs of energy production and metabolic processes, forming dynamic networks to regulate

These authors contributed equally: Wei Liu, Xiuying Duan

Edited by E. Baehrecke

Supplementary information The online version of this article (https:// doi.org/10.1038/s41418-019-0482-7) contains supplementary material, which is available to authorized users.

$\triangle$ Chao Tong

ctong@zju.edu.cn

1 The Second Affiliated Hospital, School of Medicine, Zhejiang University, Zhejiang 310009 Hangzhou, China

2 MOE Key Laboratory for Biosystems Homeostasis and Protection and Innovation Center for Cell Signaling Network, Life Sciences Institute, Zhejiang University, Zhejiang 310058 Hangzhou, China

3 Institute of Aging Research, Hangzhou Normal University, Hangzhou 311121, China

4 National Institute of Infectious Diseases and Vaccinology, National Health Research Institutes, Zhunan, Taiwan cell proliferation and cell death. Mitochondrial dysfunction leads to multiple human diseases, including neurodegeneration, and understanding the functions of disease-related molecules inside mitochondria is key to dissecting the mechanisms of these diseases and developing potential treatments [1]. The MFN1/2 [2] and OPA1 proteins are large GTPases that regulate the fusion of the outer and inner mitochondrial membranes, respectively [3]. Mutations in MFN2 lead to Charcot-Marie-Tooth neuropathy while mutations of OPA1 result in optic atrophy [4]. Defective mitochondrial fusion leads to the destruction of the mitochondrial network and mitochondrial dysfunction [5].

The CHCHD proteins belong to a family of nucleusencoded mitochondrial proteins that contain at least one coil-coil helix and coil-coil helix $(\mathrm{CHCH})$ domain [6]. The $\mathrm{CHCH}$ domain contains two CX9C motifs, each having two cysteine residues separated by nine amino acids. The cysteines form disulfide bonds that stabilize the structure of the $\mathrm{CHCH}$ domain. There are nine $\mathrm{CHCHD}$ proteins in mammals. Among them, CHCHD2 and CHCHD10 are closely related and mutations in the genes encoding these proteins lead to neurodegenerative diseases [7]. Multiple CHCHD1O genetic variants have been identified in patients 
with amyotrophic lateral sclerosis (ALS) and/or frontotemporal lobar degeneration (FTD) [8]. In addition, CHCHD10 variants have also been associated with lateonset spinal muscular atrophy (SMA), Charcot-Marie-Tooth neuropathy (CMT2), and autosomal dominant mitochondrial myopathy (IMMD) [9, 10]. In fibroblasts and in the muscle tissue of these patients, mitochondria are fragmented and the cristae have defective structures [8]. Moreover, mitochondrial respiration is reduced in these patients and deletions in mtDNA have also been reported [2]

It has been suggested that CHCHD10 is a component of the MICOS complex that is required for mitochondrial cristae formation [11-13]. Variants of CHCHD2 have been associated with Parkinson's disease (PD) [14]. The $\mathrm{CHCHD} 2$ protein functions both as a mitochondrial protein and a transcription factor $[15,16]$. Loss of $\mathrm{CHCHD} 2$ function leads to impaired mitochondrial respiration and energy production [17]. CHCHD2 was also reported to inhibit apoptosis through regulating Bax activation [18]. The Chchd2 gene is the fly homolog of both $C H C H D 10$ and CHCHD2, and loss of Chchd2 in the fly is reported to result in reduced motility and life span [19]. Furthermore, mitochondrial cristae are abnormal in the muscle tissue of the Chchd2 mutants [19]. In this study, we found that the protein encoded by Chchd2 interacts with mitochondrial protein P32 to modulate Opal levels and regulate mitochondrial fusion and cristae morphology.

\section{Results}

\section{Loss of Chchd2 leads to muscle mitochondrial defects}

To examine the physiological role of Chchd2, we generated Chchd2 knockout flies by CRISPR/Cas9 (Fig. 1a). Multiple alleles with different indels were recovered, all leading to premature termination of translation and presumably loss of protein activity. Although the resulting Chchd2 mutants were viable and fertile, adult animals exhibited climbing defects. Since all the mutants showed similar phenotypes, we selected one line, Chchd $2^{8-8}$, for detailed analysis. Quantitative PCR results indicated that $C h c h d 2^{8-8}$ mutant animals have reduced Chchd 2 mRNA expression, suggesting the nonsense-mediated mRNA decay that led to the reduction of the transcript (Fig. 1a). Compared to wild type control animals, the $C h c h d 2^{8-8}$ CRISPR mutant flies first showed climbing defects at 2 days of age and these defects were progressively enhanced with age (Fig. 1b). We then used transmission electron microscopy (TEM) to examine the flight muscles of both wild-type and $C h c h d 2^{8-8}$ flies after collecting thoraxes from 2-day-old and 30-day-old animals. In both young and old wild-type flies, mitochondria with dense cristae were packed normally between muscle fibers (Fig. 1c, c', j). However, the flight muscles of the 2-day-old $C h c h d 2^{8-8}$ mutant occasionally presented mitochondria with a reduced number of cristae (Fig. 1d, j), while the number of mitochondria with abnormal crista structures increased markedly in the flight muscles of the 30-day-old $C h c h d 2^{8-8}$ flies (Fig. 1d', j). Expression of UAS-Chchd2 driven by daughterless-Gal4 (da-Gal4) in the Chchd $2^{8-8}$ mutant flies rescued the flight muscle mitochondrial defects in both the young and old flies (Fig. 1e, e', j), indicating that Chchd2 is indeed required for mitochondrial structural integrity in muscles.

CHCHD2 and CHCHD10 are the mammalian homologs of Chchd2, and mutations in these genes are associated with Parkinson's disease, ALS, FTD, and SMA (Fig. 1f). We generated transgenic flies expressing UAS-transgenes mimicking the disease-related mutations by altering the corresponding amino acid in fly Chchd2-PA (Fig. 1f) and drove their expression with da-Gal4 in the $C h c h d 2^{8-8}$ mutant flies. The expression of $C h c h d 2^{S 81 L}$ and Chchd $2^{G 88 V}$ could not rescue the muscle mitochondrial defects in $C h c h d 2^{8-8}$ mutant flies, indicating that these disease-mimicking mutations affect normal Chchd2 functions (Fig. 1g, g', h, h', j). However, expressing $C h c h d 2^{K 164 Q}$ could rescue the muscle mitochondrial defects in $C h c h d 2^{8-8}$ mutants, suggesting that this point mutation does not severely affect the function of Chchd2 in muscles (Fig. 1i, i', j).

We also examined whether the wild type and the mutant forms of Chchd 2 could rescue the climbing defects in Chchd $2^{8-8}$. The expression of wild type Chchd 2 rescued the climbing defects in both young and old $C h c h d 2^{8-8}$ animals. Although all the mutant forms rescued the early climbing defects in the Chchd $2^{8-8}$ mutants, none of the mutant forms could rescue the climbing ability in the 30 days old animals (Fig. 1b), suggesting that all the mutant forms were not fully functional. The expression of Chchd2 $2^{S 81 L}$ and $C h c h d 2^{G 88 V}$ slightly enhanced the muscle mitochondrial defects in Chchd $2^{8-8}$ mutant flies, suggesting that these mutant forms might have dominant effects or even confered some neomorph effects. To test this, we also expressed the mutant forms Chchd 2 in the wild type background and examined the muscle mitochondrial morphology in the 2-day-old and 30-day-old animals by TEM. Indeed, the expression of Chchd2 $2^{S 81 L}$ and Chchd $2^{G 88 V}$ but not Chchd2 $2^{\text {164Q }}$ led to mild muscle mitochondrial defects in the aged animals (Fig. S1).

The expression patterns of the Chchd $2^{\mathrm{S} 81 \mathrm{~L}}$, Chchd2 ${ }^{\mathrm{G} 88 \mathrm{~V}}$, and Chchd $2^{\mathrm{K} 164 \mathrm{Q}}$ proteins were examined in the fly fat body tissues, and the results indicated that all these proteins localized to mitochondria like that seen in the wild-type controls (Fig. 1k). This suggests that these point mutations do not change Chchd 2 protein localization. 


\section{A}

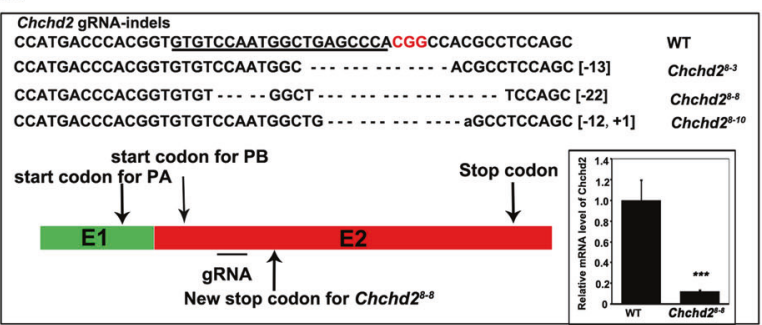

B
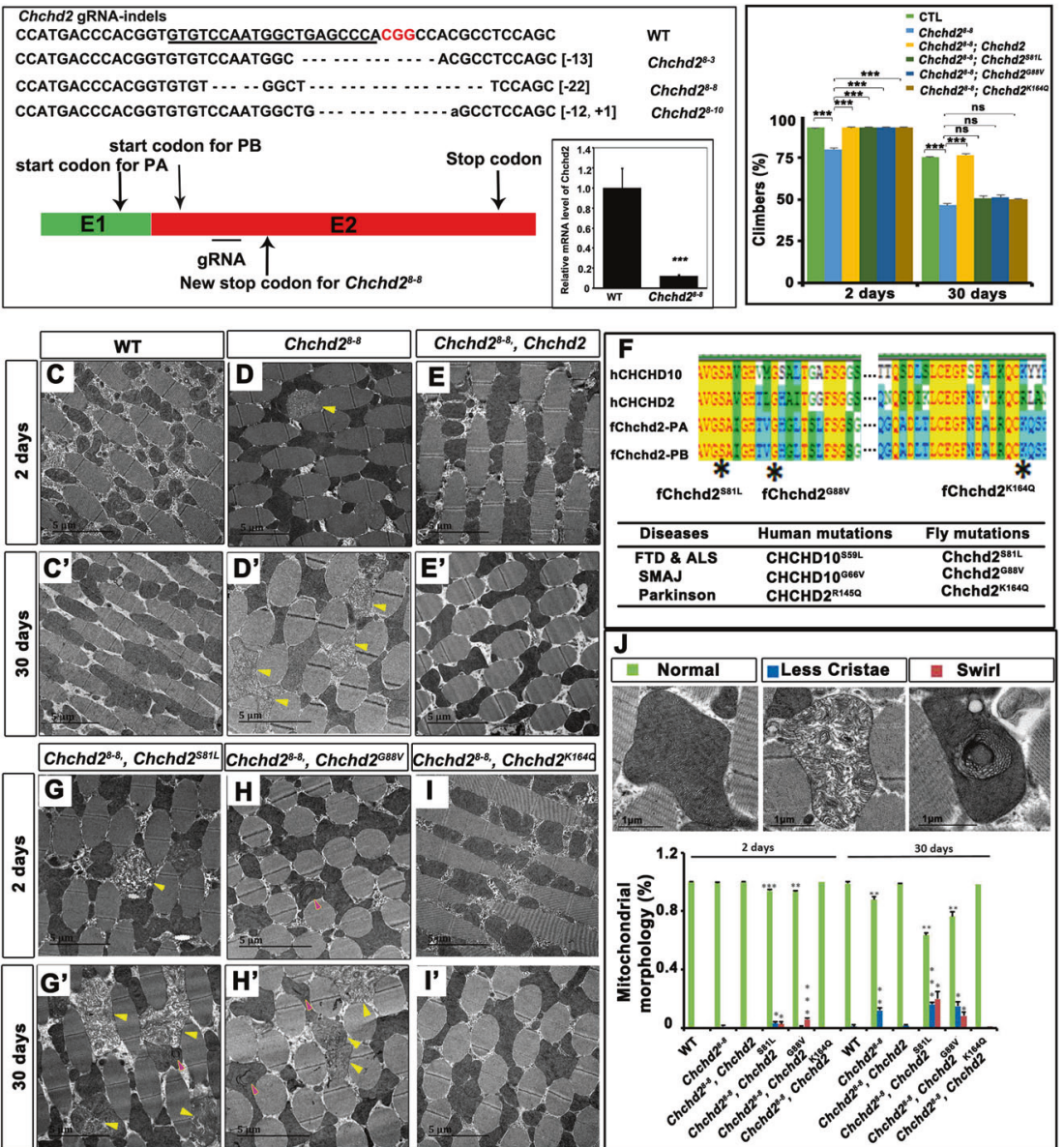

K
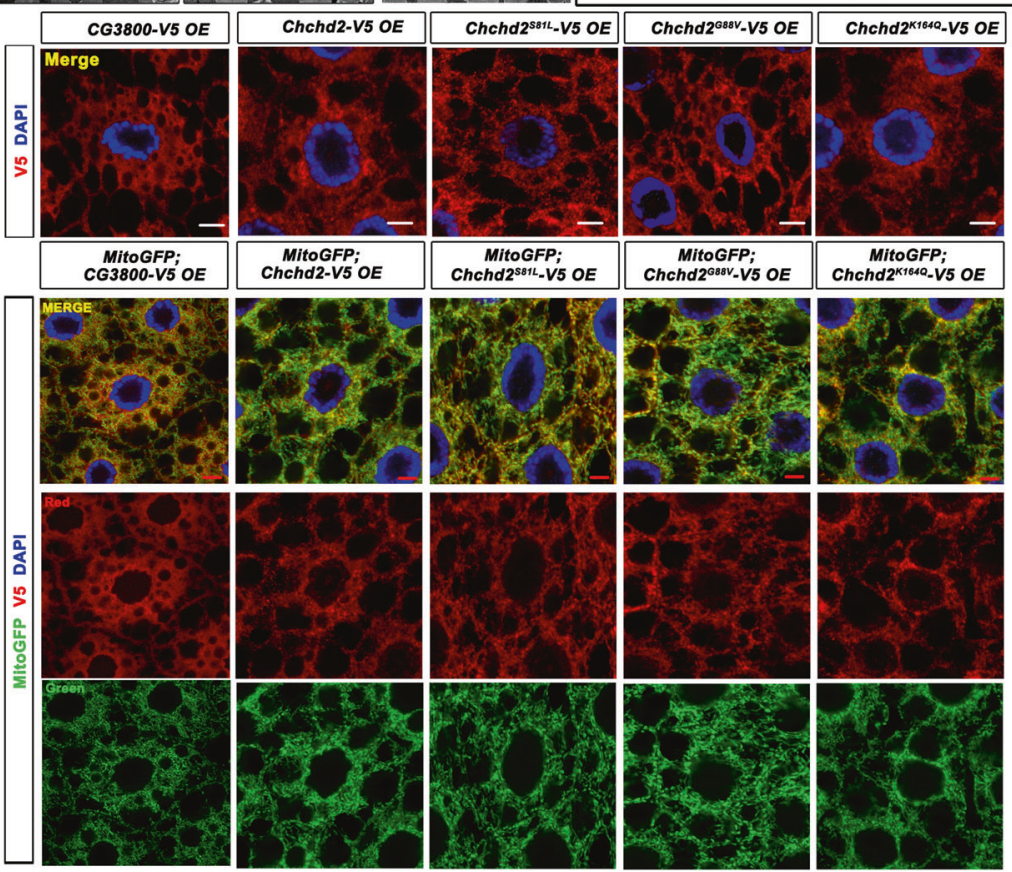
Fig. 1 Loss of Chchd2 leads to muscle mitochondrial defects. A scheme to show the CRISPR/ Cas9 mediated knock out strategy. gRNA target region (the underlined nucleotides), the Chchd 2 indel mutants generated in this study, and the predicted Chchd 2 proteins produced in wild type and $C h c h d 2^{8-8}$ animals were shown. The relative mRNA level of the $C h c h d 2^{8-8}$ mutants was greatly reduced. $* * * p<$ 0.001 . b $C h c h d 2^{8-8}$ adult flies exhibited progressively worsen climbing defects. The overexpression of Chchd 2 rescued the climbing defects of Chchd $2^{8-8}$ mutants. The overexpression of Chchd $2^{\mathrm{K} 164 \mathrm{Q}}, \mathrm{Chchd} 2^{\mathrm{S} 81 \mathrm{~L}}$, or Chchd $2^{\mathrm{G} 88 \mathrm{~V}}$ rescued the climbing defects in 2-day-old but not 30day-old $C h c h d 2^{8-8}$ flies. $n=6$ groups from 60 flies of each genotype have been analyzed. The data were presented as mean + SEM. ns: not significant; ${ }^{* * *} p<0.001$. $\mathbf{c}-\mathbf{i}$ ' The TEM of flight muscle thin sections from the young and aged animals with indicated genotypes. Chchd2 $2^{8-8}$ animals have defective mitochondria with less cristae in the flight muscles. (indicated by yellow arrows) (d and $\mathbf{d}^{\prime}$ ), which could be rescued by the over expression of wild type Chchd2 (e and $\left.\mathbf{e}^{\prime}\right)$ or Chchd $2^{\mathrm{K} 164 \mathrm{Q}}\left(\mathbf{i}, \mathbf{i}\right.$ '), but not by the expression of Chchd $2^{\mathrm{S} 81 \mathrm{~L}}\left(\mathbf{g}\right.$ and $\left.\mathbf{g}^{\prime}\right)$ or Chchd $2^{\mathrm{G} 88 \mathrm{~V}}$ (h and h'). The mitochondria with less cristae were indicated with yellow arrows and the mitochondria with the curling cristae were indicated with pink arrows. f The alignments of the protein sequences of human CHCHD10, human CHCHD10, and two isoforms (PA and $\mathrm{PB}$ ) of fly Chchd 2 to show the conserved amino acids that were identified in the patients with indicated diseases. $\mathbf{j}$ The statistics of proportions of normal and defective mitochondria in the flight muscle of the animals with indicated genotypes. The typical mitochondrial morphology was shown at the top of the panel. $n=4-6$ images from 3 flies of each genotype have been analyzed. The data were presented as mean + SEM. $* p<0.05 ; * * p<0.01 ; * * * p<0.001$. $\mathbf{k}$ Both the wild type and mutant forms of Chchd2 (red) were colocalized with mitoGFP (green) when they were co-expressed in fly fat body. The patterns of Chchd 2 and the mutant form of Chchd 2 were same when they were expressed alone (Top row) or together with mitoGFP (bottom 3 rows). The split channels of red and green were show to indicate the patterns of mitoGFP and V5 tagged different form of Chchd2, respectively. The genotype for each image was show on the top. OE: overexpression. As control, V5 tagged cytosolic protein CG3800 were over expressed in the fat body. Nuclei were marked by DAPI staining (blue). The bar indicates $5 \mu \mathrm{m}$.

\section{Chchd2 is required for neuronal integrity and loss of Pink1 or Park enhances Chchd $2^{8-8}$-associated neuronal defects}

Since several $\mathrm{CHCHD}$-related and $\mathrm{CHCHD10}$-related diseases are neurodegenerative, we investigated whether Chchd $2^{8-8}$ mutant flies exhibit neurodegeneration phenotypes in addition to the observed muscle defects. For this, we examined the photoreceptor cells in the retina, a valuable system that allows the investigation of neurodegeneration in a relatively quantitative manner. The heads of young ( 2 days old) and old (30 days old) adult flies were collected for TEM analysis (Fig. 2a-d). The eyes in 2-dayold Chchd2 mutant flies showed no obvious morphological defect (Fig. 2b, d). However, loss of rhabdomeres was observed in numerous lamina cartridges in the eyes of the 30-day-old mutants (Fig. 2b', d). To confirm that the degeneration was due to the loss of Chchd2, we used daughterless-Gal4 that expresses Gal4 ubiquitously to drive $C h c h d 2$ expression in the $C h c h d 2^{8-8}$ mutants. The
daughterless-Gal4-driven expression of Chchd2 could fully rescue the degeneration phenotype of the photoreceptor cells (Fig. 2c, d) in the Chchd $2^{8-8}$ mutants, indicating that Chchd2 is the gene responsible for the eye degeneration phenotype.

In addition to eye degeneration, we also investigated whether dopaminergic (DA) neurons were affected in Chchd $2^{8-8}$ mutant flies. The DA neurons are clustered in six different groups (PPM1, PPM2, PPM3, PPL1, PPL2c, and PPL2ab) in each hemisphere of the fly brain [20]. We used anti-tyrosine hydroxylase (TH) antibody staining to label the DA neurons. Compared to the wild-type controls (Fig. 2e, g), 30-day-old Chchd $2^{8-8}$ mutant flies (Fig. 2f, g) showed no statistically significant reduction of the number of DA neurons, indicating that $C h c h d 2$ is not required to maintain DA neuron integrity in the fly central nervous system (CNS).

We also examined whether the neurodegeneration defects in $C h c h d 2^{8-8}$ mutant flies could be enhanced by the loss of Pinkl or Park, the fly homologs of two human Parkinson's disease-related genes (Fig. 2h-n) [21]. Knockdown of Pinkl or park by RNAi elicited only a small loss of photoreceptor cells in the eyes of the older flies (30 days old) (Fig. 2j', l', n'). Similarly, Chchd $2^{8-8}$ mutant flies also presented mild degeneration of photoreceptor cells (Fig. I, I', N, N'). However, knocking down Pinkl or park in $C h c h d 2^{8-8}$ mutant flies resulted in a considerably stronger photoreceptor cell degeneration phenotype in the older animals (Fig. 2k', m', n').

\section{Loss of Chchd2 leads to fragmentation of mitochondria}

Chchd2 encodes two polypeptides, namely, Chchd2-PA and Chchd2-PB (Fig. 3a). The Chchd2-PB isoform is shorter than Chchd2-PA due to the absence of a potential mitochondrial targeting sequence (MTS). To investigate whether they localize to mitochondria, we overexpressed both isoforms in cultured Drosophila S2 cells and larvae fat body tissues, together with the mitochondrial marker MitoGFP. Both Chchd2-PA and Chchd2-PB colocalized with MitoGFP (Fig. 3b-g), indicating that the Chchd2 proteins indeed localize to mitochondria and that the potential MTS was not essential for the mitochondrial targeting of Chchd 2 proteins. To examine whether loss of Chchd2 affects mitochondrial fission and fusion, we examined mitochondrial morphology in fly fat body tissues (Fig. 3h-s). The fly fat body consists of a single layer of large cells, making this an ideal system to study organelle morphology. In Chchd $2^{8-8}$ mutant flies, early third instar larvae fat body tissues exhibited fragmented mitochondria (Fig. 3m, m'). In addition, the average length of the mitochondria was reduced compared to the wild-type 

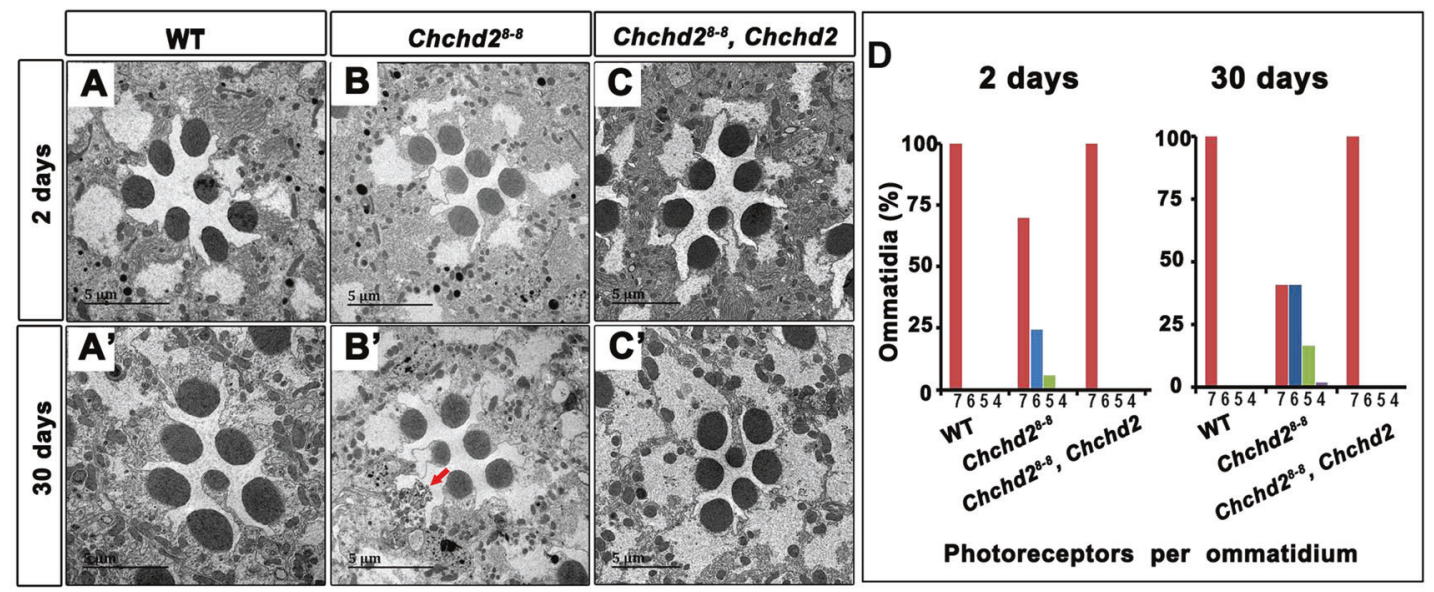
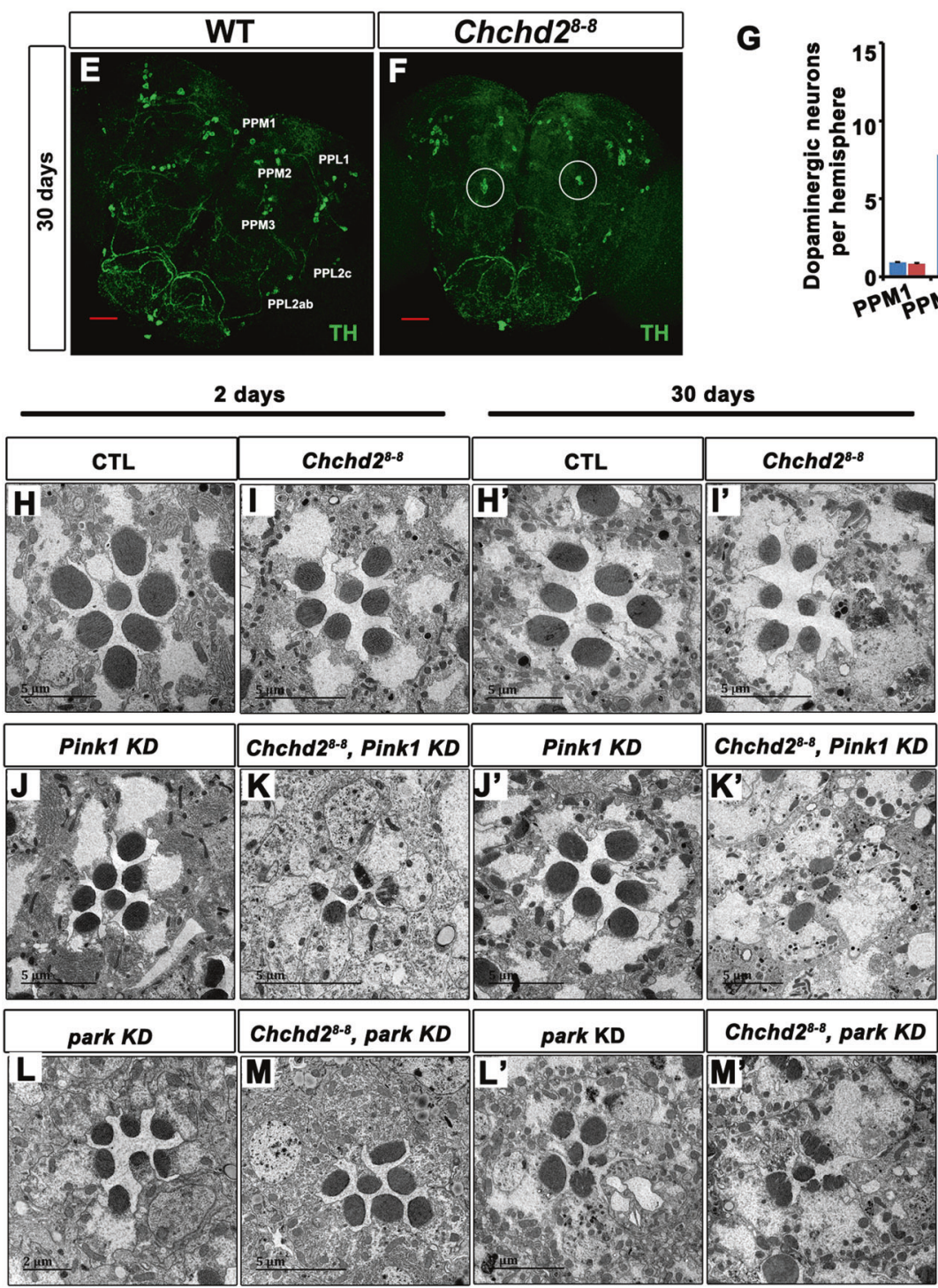

G

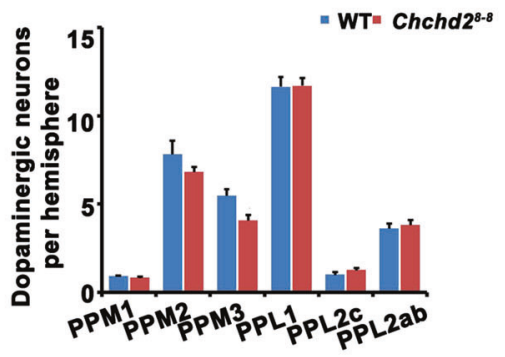

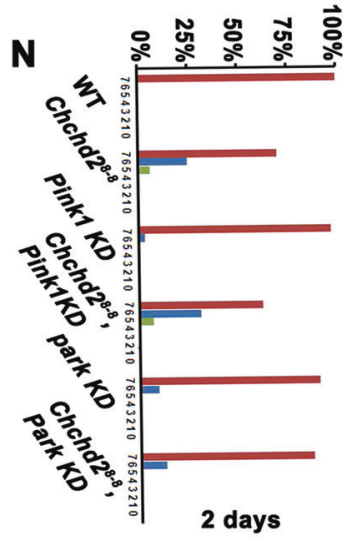

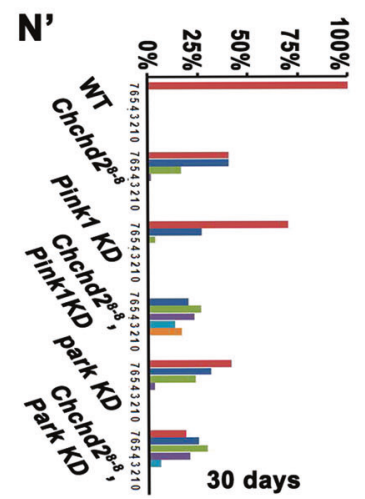

control tissues (Fig. 3r), suggesting that the mitochondria in Chchd $2^{8-8}$ mutant flies may have either reduced fusion or excessive fission.
To further determine the role of Chchd2 in mitochondrial fission and fusion processes, we overexpressed or knocked down key molecules that regulate mitochondrial dynamics 
Fig. 2 Chchd2 is required for neuronal integrity. a-c' $C h c h d 2^{8-8}$ mutants showed reduced number of rhabdomeres in the retina of the aged flies (b', indicated by the red arrow), which could be rescued by overexpression of the wild type Chchd2. d The bars indicated the percentage of ommatidia with indicated numbers of rhabdomeres in the animals with different genotypes. $n=38-71$ ommatidia from 3 eyes of each genotype have been analyzed. e-g The numbers of TH neurons in the $C h c h d 2^{8-8}$ mutants were comparable to that of the wild type animals. Anti-TH staining (green) indicates the TH neurons in the 30 days old fly brains with indicated genotypes. The bar indicates $50 \mu \mathrm{m} . \mathbf{g}$ is the statistics of the TH neuron numbers in the indicated regions in wild type (WT) and $C h c h d 2^{8-8}$ mutants. $n=12-14$ heads have been analyzed. The data were presented as mean + SEM. WT vs. Chchd $2^{8-8}$, PPM1 $p=0.9129$, PPM2 $p=0.2319$, PPM3 $p=0.0099$, PPL1 $p=0.9358$, PPL2c $p=0.2329$. PPL2ab $p=0.6289$. ns, nonsignificant, $p>0.0083$, one way ANOVA with post hoc Bonferroni's test. h-m' Pinkl and park knock down (KD) enhanced the eye degeneration phenotypes in Chchd $2^{8-8}$ mutants. n and n' The bars indicated the percentage of ommatidia with indicated numbers of rhabdomeres in the animals of different genotypes. $n=22-89$ ommatidia from 3 eyes of each genotype have been analyzed.

in both wild-type and $C h c h d 2^{8-8}$ mutant fat body tissues and assessed mitochondrial morphology through analysis of MitoGFP fluorescence patterns. Marf is the fly homolog of $M F N 1 / 2$ that regulates the fusion of the outer mitochondrial membrane [22], while Drp1 is a dynamin-like GTPase that mediates mitochondrial fission [23]. Overexpression of Marf in wild-type fat body tissues resulted in the formation of large MitoGFP blobs (Fig. 3i, i', s). However, no MitoGFP blob was observed when Marf was overexpressed in $C h c h d 2^{8-8}$ mutant fat body tissues (Fig. 3n, n', s). Loss of Drpl in wild-type fat body tissues led to Large MitoGFP blob formation. Knockdown of Drpl in Chchd2 ${ }^{8-8}$ tissues resulted in a greatly reduced number of large MitoGFP blobs (Fig. 3j, j', o, o', s). Overexpression of Drpl resulted in small, fragmented mitochondria in fat body tissues (Fig. 3k, k', r). However, this phenotype was not further enhanced by loss of Chchd2 (Fig. 3p, p', r). Opa1 mediates the fusion of the inner mitochondrial membranes [24, 25]. Targeting Opa1 by RNAi in the wild-type fat body tissues led to fragmented mitochondria (Fig. 31, 1', r), which was aggravated by the loss of Chchd2 (Fig. 3q, q', r). These data suggest that Chchd2 is indeed required for regulation of mitochondrial dynamics.

\section{Chchd2 modulates the levels of Opa1}

To understand how the loss of Chchd2 affects mitochondrial morphology, we examined the expression of the genes encoding key elements of the mitochondrial fusion-fission machinery in $C h c h d 2^{8-8}$ mutants by real-time PCR. The results showed that expression of Opal was decreased in the $C h c h d 2^{8-8}$ mutants, whereas the expression of Marf and Drpl was largely unaffected (Fig. 4a). We further examined the protein level of Opa1. Because there is no good antibody available for fly Opa1, the levels of Opa1 were evaluated instead in a CRISPR/Cas9-mediated Opal-3HA knock-in line. We collected adult thorax muscles from Opal-3HA or Chchd2 ${ }^{8-8}$; Opal-3HA flies and measured the levels of HA-tagged Opa1 by western blot. Loss of Chchd2 significantly reduced the level of Opa1 (Fig. 4b, b'). Moreover, when we co-overexpressed Chchd2 and 3HAtagged Opa1 in S2 cells, the Opa1-3HA level increased with increasing Chchd2 expression (Fig. 4c, c'), even though the same concentration of Opa1-3HA DNA was transfected. Since we used the UAS-Gal4 system to express Opa1-3HA, the change recorded in the levels of Opa1-3HA was likely due to post-transcriptional regulation. These results indicate that Chchd2 may be involved in stabilizing Opa1. We then tested whether Chchd 2 could bind to Opa1 by coimmunoprecipitation of the proteins overexpressed in $\mathrm{S} 2$ cells. We did not detect any interaction between Chchd2 and Opa1 (Fig. 4d), suggesting that Chchd2 may regulate Opa1 levels indirectly.

Studies using mammalian cells have indicated that OPA1 can be processed by YME1L and OMA1 [26-28]. Although there is a fly homolog of human $Y M E 1 L$, there is no fly homolog of human OMA1. Therefore, we tested whether fly YME1L could also process Opa1 and regulate its activity. We expressed FLAG-tagged Opa1 in S2 cells, with or without HA-tagged YME1L, and examined Opa1-FLAG expression by western blot. Although the western blot pattern of the Opa1 bands did not change with expression of HA-tagged YME1L, the level of the Opa1 protein was significantly reduced (Fig. 4e, e'). However, when Chchd2 was co-expressed with YME1L and Opa1, the YME1Linduced reduction in Opa1 levels was reversed (Fig. 4e, e'), suggesting that Chchd2 might regulate Opa1 levels by modulating YME1L activity. We further tested whether Chchd 2 could interact with YME1L by expressing YME1L and Chchd2 in S2 cells and performing immunoprecipitation (IP) experiments. However, no interaction was detected between Chchd2 and YME1L (Fig. 4f), suggesting that Chchd 2 might regulate YME1L activity indirectly.

Human $\mathrm{CHCHD} 2$ and $\mathrm{CHCHD} 10$ have both been reported to interact with $\mathrm{C} 1 \mathrm{QBP}$, a chaperone-like mitochondrial protein [29, 30]. In a mass spectrometry analysis of proteins immunoprecipitated with an anti-human CHCHD2 antibody from Hela cells, we also identified numerous peptides from C1QBP (Fig. 4g, Table S1). To confirm the interaction, we expressed V5-tagged C1QBP in Hela cells and performed the IP assay using an anti-V5 antibody. We found that endogenous CHCHD2 coprecipitated with C1QBP-V5 (Fig. 4h). The P32 protein is the Drosophila homolog of C1QBP [31], and to test whether P32 interacts with fly Chchd2, we overexpressed P32HA and Chchd2-V5 in S2 cells and carried out IP experiments. Consistent with the data from mammalian cells, we found that Chchd2-V5 co-precipitated with P32-HA 

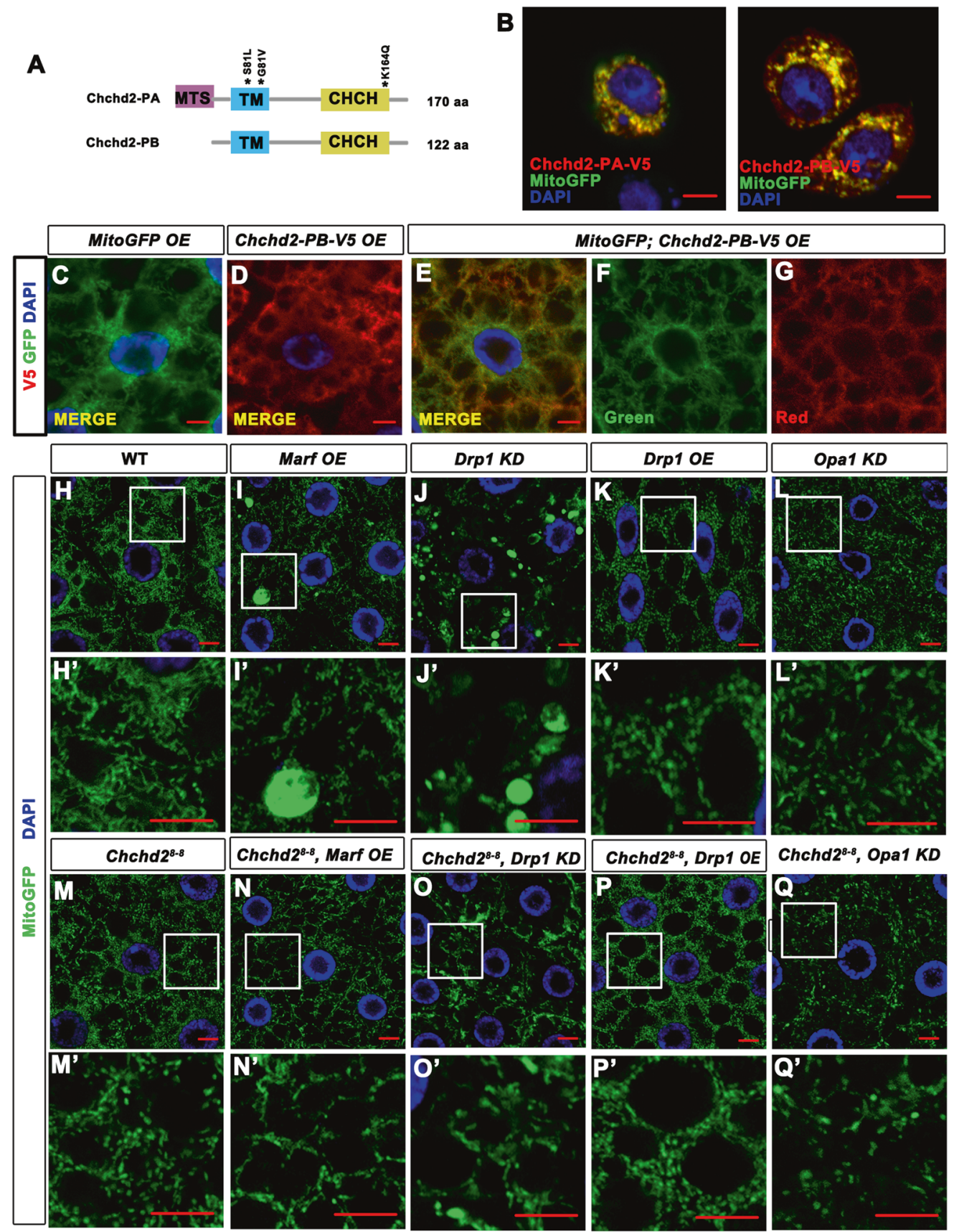

Chchd2 $2^{8-8}$, Drp1 KD Chchd28-8, Drp1 OE Chchd2 ${ }^{8-8}$, Opa1 KD

$\mathbf{R}$

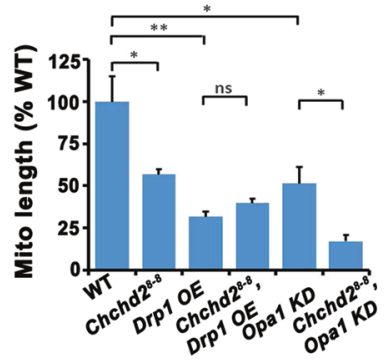

(Fig. 4h). We also tested whether Chchd2 ${ }^{\text {S81L }}$ and Chchd $2^{\mathrm{G} 88 \mathrm{~V}}$ could interact with P32. Co-precipitation assay indicated that all these mutant forms of Chchd 2 interacted with P32, suggesting that the malfunctions of these mutant forms of Chchd2 is not due to the lack of interaction with P32 (Fig. S2A). 
Fig. 3 The mitochondria were fragmented in Chchd28-8 mutants. a A diagram showed the domain organization in two isoforms of Chchd2 proteins. MTS: mitochondrial targeting sequence. TM: transmembrane domain. $\mathrm{CHCH}$ : $\mathrm{CHCH}$ motif. b Both PA-Chchd 2 and PB-Chchd2 (red) are co-localized with MitoGFP (green) when they were overexpressed in S2 cells. The bar indicates $5 \mu \mathrm{m}$. c-g PBChchd 2 co-localized with MitoGFP when it was overexpressed in fat body tissues. c Fat body cells with MitoGFP (green) overexpression. d Fat body cells with PB-Chchd2 (red) overexpression. e, f Fat body cells with MitoGFP (green) and PB-Chchd2 (red) co-expressed. g and $\mathbf{f}$ are split channels of $\mathbf{e}$. $\mathbf{h}-\mathbf{q}$ ' The patterns of MitoGFP (green) in the fat body tissues of early third instar larvae with indicated genotypes. h'-l' are the high magnification images of the boxed regions in $\mathbf{h}-\mathbf{l}$. Chchd $2^{8-8}$ fat body tissues have fragmented mitochondria (comparing $\mathbf{m}, \mathbf{m}$ ' with $\mathbf{h}, \mathbf{h}$ '). Over-expression (OE) of Marf (i and $\mathbf{i}$ ') or RNAi knock down (KD) Drp1 (j and $\mathbf{j}^{\prime}$ ) led to swollen mitochondria exhibit as large MitoGFP puncta, which are disappeared when $C h c h d 2$ was lost (n, n', o, and o'). Drp1OE (k, k') and Opal KD (l, l') led to fragmented mitochondria. Loss of Chchd2 did not further enhance Drp1OE phenotypes (p, p'), but further reduced mitochondrial size of the Opa1 KD animals (q, q'). The bar indicates $5 \mu \mathrm{m}$. $\mathbf{r}$, s The statistics of mitochondrial length and the MitoGFP puncta size of the animals with indicated genotypes. $n=4-7$ images from 4 to 7 flies of each genotype have been analyzed. The data were presented as mean + SEM. ns, not significant; ${ }^{*} p<0.05 ;{ }^{* *} p<0.01 ; * * *<<0.001$.

\section{Chchd2 interacts with P32 to regulate mitochondrial morphology}

We further investigated whether the interaction between Chchd 2 and P32 could affect mitochondrial morphology (Fig. 5a-e). In fat body tissues, knocking down $p 32$ did not result in obvious changes in mitochondrial morphology (Fig. 5c, c', e). Surprisingly, reducing p32 expression in the fat body tissues of $C h c h d 2^{8-8}$ mutants rescued the mitochondrial fragmentation defects caused by the loss of Chchd2 (Fig. 5d, d', e). In the flight muscles of 2-day-old flies, reduced p32 expression led to mild mitochondrial defects characterized by the occasional presence of vacuolar structures inside the mitochondria (Fig. 5h, j). In 30-day-old flies, a larger number of mitochondria exhibited these vacuolar structures when p32 levels were reduced (Fig. 5h', j). Chchd2 ${ }^{8-8}$ mutant flies, especially the older ones, had defective mitochondria with fewer cristae in the flight muscles (Fig. 5g, g', j). However, when we targeted p32 by RNAi in a $C h c h d 2^{8-8}$ mutant background, the mitochondria defects were reduced compared to that observed with either p32 RNAi alone or in $C h c h d 2^{8-8}$ mutants. Most of the mitochondria in the muscle were normal and only a small fraction had fewer cristae or vacuolar structures (Fig. 5i, i', j). We further checked whether the reduction of $p 32$ in the muscle could rescue the climbing defects in $C h c h d 2^{8-8}$ mutants. Although it rescued in the 2-day-old animals, the climbing ability has not been improved in the 30-day-old Chchd $2^{8-8}$ mutants with $p 32$ knockdown (Fig. S2B). We also examined whether $p 32$ RNAi could rescue the photoreceptor cell degeneration. p32 RNAi alone in the eyes led to mild photoreceptor cell degeneration. Knocking down P32 slightly increased the ratio of the intact photoreceptor cells in the aged $C h c h d 2^{8-8}$ mutants (Fig. S2C-E). These data suggesting that the reduction of p32 could compensate some defects caused by the loss of Chchd2.

Since the level of Opa1 was reduced in $C h c h d 2^{8-8}$ mutants, we then examined whether reduced levels of P32 affect the levels of Opa1 in wild-type and $C h c h d 2^{8-8}$ mutants. Opal-3HA knock-in alleles were introduced into the $\operatorname{Chchd2}^{8-8}$ mutant flies, p32 knockdown flies, or Chchd $2^{8-8}$ mutant with $p 32$ knockdown. The fat body tissues of third instar larvae were collected for western blot analysis. Consistent with the mitochondrial fragmentation phenotypes, the Opal level was reduced in the Chchd $2^{8-8}$ mutant flies. Targeting $p 32$ by RNAi increased Opa1 levels in both wild-type and $C h c h d 2^{8-8}$ mutants, but the levels in the $C h c h d 2^{8-8}$ mutants were marginally higher than those in the wild type animals (Fig. 5k, k'). We then tested whether P32 forms a complex with Opa1 in S2 cells using IP, but no interaction was detected (Fig. 51). We also tested whether P32 could interact with YME1L. We co-expressed V5tagged P32 and HA-tagged YME1L in S2 cells and pulled down P32 with an anti-V5 antibody. We found that YME1L co-precipitated with P32 (Fig. 5m). Moreover, we also found that co-expressing YME1L and Opa1 reduced the level of Opa1, which was then further reduced when P32 was co-expressed with both YME1L and Opa1 (Fig. 5n, n'). This suggests that the interaction between YME1L and P32 may enhance YME1L activity.

Since both Chchd2 and YME1L interact with P32, we hypothesized that Chchd 2 might modulate YME1L activity by competing with YME1L for P32 binding. We coexpressed YME1L and P32 with or without Chchd2 in S2 cells and performed IP for P32 to detect YME1L. Indeed, expressing Chchd2 decreased the amount of YME1L coprecipitated with P32 (Fig. 5o, o').

\section{Discussion}

Although it was named after one of its mammalian homologs, CHCHD2, fly Chchd2 is also the homolog of human $\mathrm{CHCHD} 10$. Both $\mathrm{CHCHD} 2$ and $\mathrm{CHCHD} 10$ are human disease-related genes. To date, no Chchd2 knockout mouse has been reported. However, homozygous Chchd10 knockout mice are viable and grossly normal [30]. The molecular mechanisms underlying the diseases caused by the mutations in $C H C H D 2$ and CHCHD1O are still elusive. In this study, we generated fly CRISPR/Cas9 Chchd2 mutants and found that loss of Chchd2 resulted in muscle defects and neurodegeneration. The fly Chchd 2 presenting with mutations that mimic the disease-related ones did not rescue some of the 

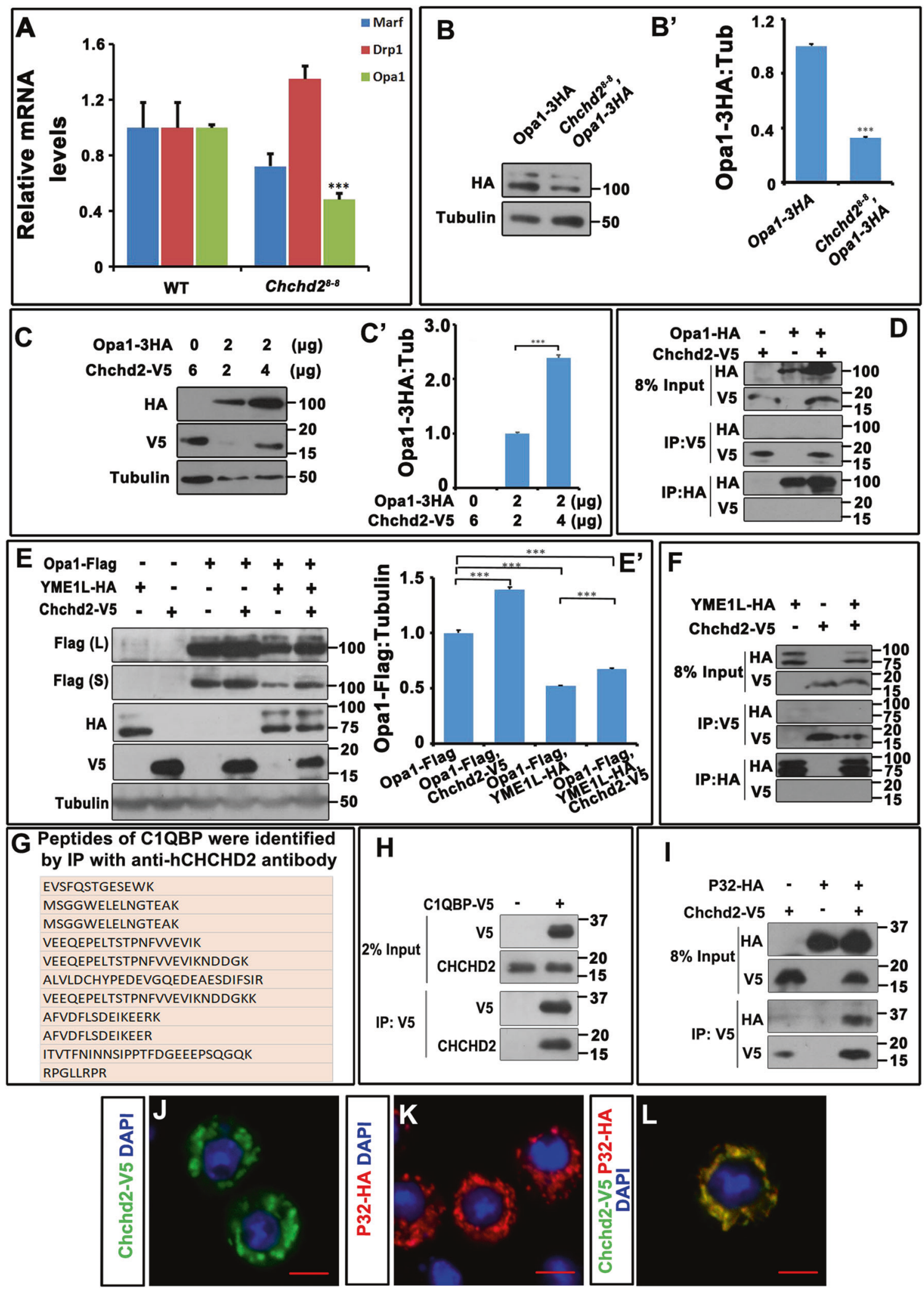

Chchd2 mutant phenotypes, suggesting that these conserved amino acids are critical for Chchd 2 protein function. Therefore, this study provided a good example of using the fly model to study human diseases, especially when other animal models are lacking or fail to mimic disease phenotypes. 
4 Fig. 4 Chchd2 modulates the levels of Opa1. The relative mRNA levels of Marf, Drp1, and Opa1 in wild type (WT) and Chchd2 ${ }^{8-8}$ mutants were measured by real-time PCR. Opal mRNA level was significantly decreased in $C h c h d 2^{8-8}$ mutants. The experiment has been repeated 3 times. The data were presented as mean + SEM. $* * * p<$ 0.001. b, b' The protein levels of Opa1 were decreased in $C h c h d 2^{8-8}$ mutants. The endogenous levels of Opal were measured through examine the $3 \times$ HA Opal knock in animals. Tubulin levels were examined as a loading control. $\mathbf{b}$ ' is the quantification of $\mathbf{b}$. The experiment has been repeated 3 times. The data were presented as mean + SEM. $*^{* *} p<0.001$. c, $\mathbf{c}^{\prime}$ Chchd 2 overexpression stabilized Opal in a dose dependent manner. Same amount of DNA encoding $3 \times$ HA tagged Opa1 was co-expressed with increased amount of DNA encoding Chchd 2 in S2 cells. Tubulin levels were examined as a loading control. $\mathbf{c}^{\prime}$ is the quantification of $\mathbf{c}$. The experiment has been repeated 3 times. The data were presented as mean + SEM. $* * * p<$ 0.001. d Chchd 2 and Opa1 did not pull down each other in the immunoprecipitation experiments. e, e' Overexpression of YME1L reduced the levels of Opa1, whereas co-expression of Chchd 2 could counteract this effect. $\mathbf{e}$ ' is the quantification of $\mathbf{e}$. The experiment has been repeated 3 times. The data were presented as mean + SEM. ***p $<0.001$. f Chchd2 and YME1L did not pull down each other in the immunoprecipitation experiments. $\mathrm{g}$ A list of $\mathrm{C} 1 \mathrm{QBP}$ peptides that were identified by immunoprecipitation with anti-human CHCHD2 antibody followed by mass spectrometry. h Overexpressed V5-tagged C1QBP could pull down endogenous CHCHD2 in Hela cells. i V5tagged fly Chchd 2 proteins could pull down HA-tagged P32 when both were overexpressed in S2 cells. $\mathbf{j}-\mathbf{l}$ Chchd 2 and P32 were colocalized in $\mathrm{S} 2$ cells. The nuclei were marked with DAPI staining (blue). The bar indicates $5 \mu \mathrm{m}$.

The mitochondria are fragmented and have fewer cristae in the Chchd2 mutants, resembling the mitochondrial defects seen in patients with $C H C H D 10$ mutations. In Chchd2 mutant flies, both the mRNA and protein levels of Opa1 were reduced, which likely contributes to the morphological defects seen in the mitochondria since Opa1 is required for mitochondrial fusion and correct cristae morphology. Human CHCHD2 was reported to be upregulated under hypoxic stress and shuttles between mitochondria and the nucleus [15]. Nuclear-localized CHCHD2 functions as a transcription factor, regulating the transcription of genes that harbor the oxygen-responsive element [3]. Recently, CHCHD10 has also been reported to function as a transcriptional repressor in the nucleus [32]. There are two isoforms of Chchd2 in the fly. Although the protein product of transcript Chchd2-RB lacks a potential MTS in the Nterminus, both protein products localize to the mitochondria. In our study, we did not observe obvious nuclear localization, either in vivo or in vitro. However, we cannot exclude the possibility that small amount of Chchd 2 below our detection limits exists in the nuclei and functions as transcriptional regulator. It would be interesting to test whether the change observed in the level of Opal mRNA was due to the lack of Chchd 2 transcriptional activity.

In this study, we found that the Opa1 protein level was also modulated by Chchd 2 post-transcriptionally. We found that expression of fly YME1L reduces Opa1 levels. The
Chchd2 binding partner, P32, also binds to YME1L to enhance YME1L activity, while Chchd2 competes with YME1L to interact with $\mathrm{P} 32$ thereby reducing YME1L activity. It was surprising that fly YME1L reduced Opa1 levels since the YME1L mammalian homolog is required for the fusion activity of OPA1 [33]. In a mammalian study, YME1L was reported to be required for OPA1 processing at the S1 site to generate a soluble long form of OPA1 (LOPA1), which is then further cleaved by another protease, OMA1, at the L2 site to generate the short form of OPA1 (S-OPA1) [34]. The L-OPA1 form is required for mitochondrial inner membrane fusion while the S-OPA1 form has opposite activity, promoting inner membrane fission instead. Together, L-OPA1 and S-OPA1 balance mitochondrial fusion-fission processes [26, 28, 33]. The OPA1 protein is evolutionarily conserved from yeast to human. The yeast OPA1 homolog is Mgm1 and both Mgm1 and OPA1 are subjected to proteolytic processing, although the enzymes involved are different. Yeast Mgm1 is processed by the rhomboid protease Pcp1 but the human homolog of yeast Pcp1, PARL, does not cleave OPA1 [34, 35]. In flies, how OPA1 is cleaved is not well understood. Rhomboid-7, a mitochondrial rhomboid protease, is required for mitochondrial fusion and loss of Rhomboid-7 results in phenotypes similar to those observed with loss of OPA1 [36]; this indicates that fly and yeast regulate OPA1 activity via the same mechanism [37]. YME1L exists in flies, but not OMA1. Both the S1 and S2 cleavage sites are not well conserved in the fly Opa1 protein. Instead of producing functional L-OPA1, YME1L seems to reduce Opa1 levels in flies. The regulation could be direct or indirect, although the exact mechanism is unknown.

Multiple functions have been reported for the C1QBP/ P32 protein, including acting as a multifunctional chaperon to regulate kinase activity [38] and as a mRNA binding protein to facilitate mitochondrial ribosome organization [39]. Here, we found it interacted with YME1L and Chchd2 to fine tune the levels of Opa1. The detailed mechanisms are still not clear. Chchd 2 has been reported to interact with the MICOS complex to regulate mitochondrial cristae organization [13]. We did not identify any subunit of the MICOS complex in our IP and mass spectrometry experiments, suggesting that the interaction might be transient and dynamic and could not be detected under our study conditions.

Here, we propose that Chchd 2 modulates Opal levels, and consequently mitochondrial fusion, by competing with YME1L for P32 binding; as the activity of YME1L is enhanced by binding to P32, loss of Chchd2 would enhance YME1L binding to P32, thus increasing the activity of YME1L and decreasing the level of Opal and leading subsequently to mitochondrial fragmentation. Reducing the levels of P32 in Chchd2 mutants reversed the 

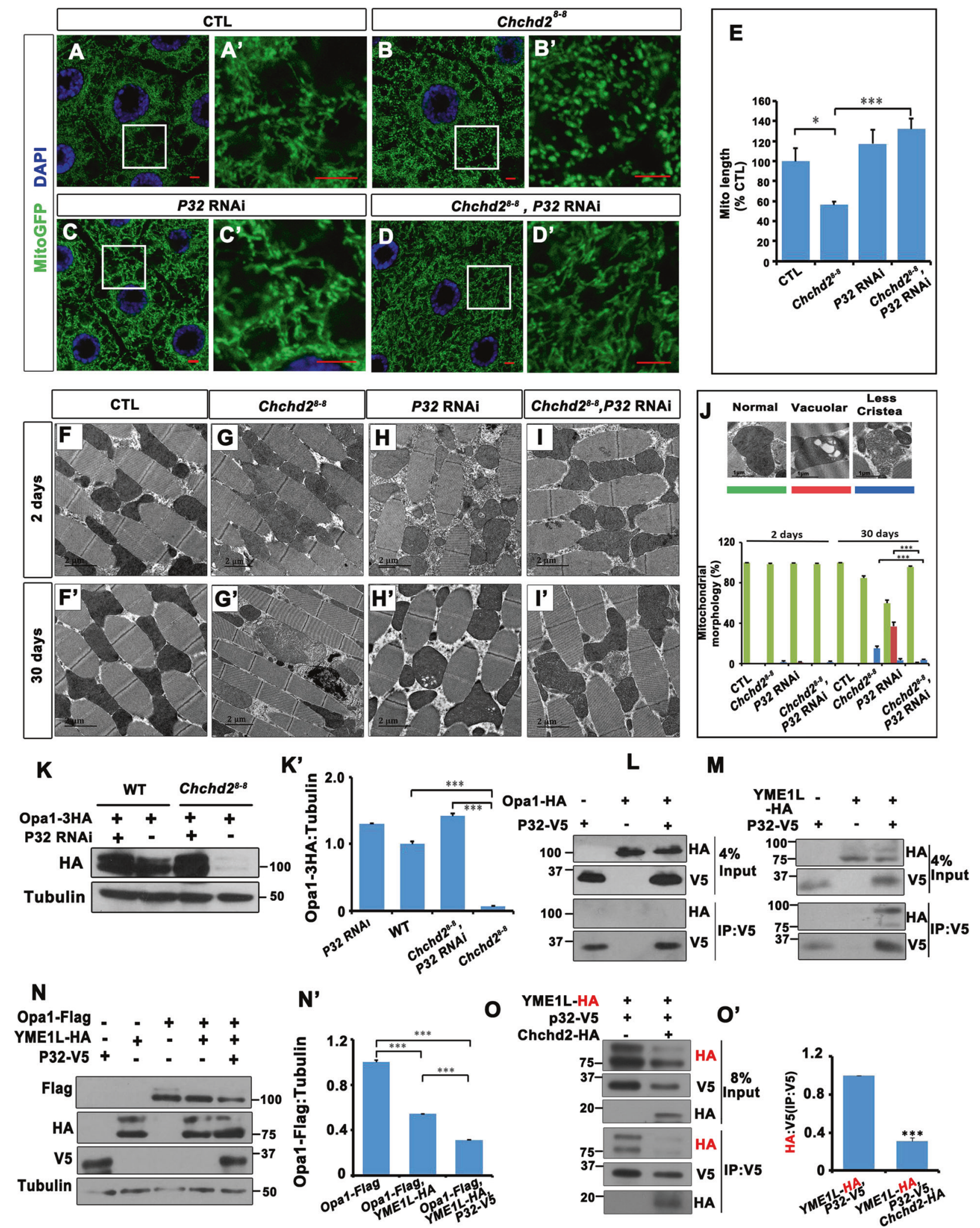

P32-associated enhancement of YME1L activity, thus rescuing some of the defects in the Chchd2 mutants (Fig. 6). In this study, we found that the diseases related form of
Chchd 2 proteins preserved the ability to bind to P32. It needs further investigation how these mutant forms of Chchd2 affect Chchd2 functions. 
Fig. 5 P32 counteract with Chchd2 to modulate mitochondrial morphology. a-e In fly fat body, loss of Chchd2 resulted in the fragmented mitochondria. The reduction of $p 32$ did not cause obvious mitochondrial morphology change, whereas reduction of $p 32$ expression in Chchd 2 mutants rescued the fragmentation in mitochondria. a', b', c', and d' are enlarged images of the boxed regions in $\mathbf{a}, \mathbf{b}, \mathbf{c}$, and d, respectively. MitoGFP (green) was used to label mitochondria and DAPI (blue) was used to mark nuclei. The bar indicates $5 \mu \mathrm{m}$. e was the quantification of relative mitochondrial length in the fat body tissues of the animals with indicated genotypes. $n=5-7$ images from 5 to 7 flies of each genotype have been analyzed. The data were presented as mean + SEM. $* p<0.05 ; * * * p<0.001$. f-j In the flight muscles of aged (30 days old) animals, loss of Chchd2 led to increased percentage of mitochondria with less cristae $(\mathbf{g}, \mathbf{j})$. When $p 32$ was knocked down, some mitochondria had vacuolar structures in the flight muscle of the 30 days old flies $(\mathbf{h}, \mathbf{j})$. The percentage of the defective mitochondria were much less in the Chchd2 mutant animals with $p 32$ knocked down than that in the Chchd 2 mutants or p32 RNAi alone animals (i', j'). The top panels of (j) were images of typical morphology of defective mitochondria and their color code in the bar figures on the bottom panel of (j). $n=4-8$ images from 3 flies of each genotype have been analyzed. The data were presented as mean + SEM. *** $p<0.001$. k, k' The knockdown of $p 32$ restored the level of Opa1 in the Chchd 2 mutant muscles. $\mathbf{k}$ ' is the quantification of $\mathbf{k}$. Endogenous levels of Opa1 were measured by using a 3HA tagged Opal knock-in allele. The experiment has been repeated 3 times. The data were presented as mean + SEM. $* * * p<0.001$. I Overexpressed V5 tagged P32 could not pull-down the overexpressed HA tagged Opa1 in S2 cells. m V5 tagged P32 could pull-down HA tagged YME1L when both were overexpressed in S2 cells. (n, n') Opa1 level was decreased when YME1L was co-expressed with Opa1. The coexpression of YME1L and P32 with Opalfurther reduced Opa1 expression level. $\mathbf{n}$ ' is the quantification of $\mathbf{n}$. The experiment has been repeated 3 times. The data were presented as mean + SEM. $* * * p<$ 0.001. o, o' Co-expression of HA tagged Chchd2 with HA tagged YME1L and V5 tagged P32 reduced the amount of YME1L immuneprecipitated with P32. $\mathbf{o}$ ' is the quantification of $\mathbf{o}$. The experiment has been repeated 3 times. The data were presented as mean + SEM. $* * * p<0.001$.

\section{Material and methods}

\section{Molecular cloning}

To generate pBluescript SK(+)-Opa1-3xHA-stop HR donor vector, the 3xHA-tag fusion L-arm of Opal flanking sequence without stop codon was generated by PCR reaction using genomic DNA of the $\mathrm{w}^{1118}$ strain as template and Opa1-stop-3xHA_up primers: UF_5'-cgggcccccctcgagGT TGCAGCAGGAATTCATATCGCTGATGGA-3', UR1_ 5'-GCCCGCATAGTCAGGAACATCGTATGGGTACT TTTCCTGATTAAGTGAATTGATGAACTC-3', UR2_5'TCCTGCATAGTCCGGGACGTCATAGGGATAGCCCG ATAGTCAGGAACATCGTATGGGTA-3', and UR3_5'AGCGTAATCTGGAACGTCATATGGATAGGATCCTG CATAGTCCGGGACGTCATAGGGATA-3'. The gRNA target site modified R-arm of Opal flanking sequence was also generated by PCR reaction using genomic DNA of the $w^{1118}$ strain as template and Opa1-stop-3xHA_down primers: DF_5'-gttccagattacgctTAGTGTGCGATGACCA
AAGACACAACACCC-3' and DR_ 5'-tggeggecgetcta gAGCAGCTAGACTAAGCGGAGGATCGGAGTGG-3'.

The agarose gel purified PCR products of L-arm and R-arm were cloned into the Aval/XbaI sites of pBluescript $\mathrm{SK}(+)$ vector using In-Fusion HD Cloning Kit (Clontech).

To generate pDCC6-Opa1-stop gRNA vector, The CRISPR target site of Opal gene for CRISPR/Cas9 recognition was designed by flyCRISPR Optimal Target Finder platform. One target site that was close to the stop codon of Opal gene had been found. Primer sets gRNA-F_5'cttcGGGTGTTGTGTCTTTGGTCA-3' and gRNA-R_5'aacTGACCAAAGACACAACACCC-3' were synthesized (Integrated DNA Technologies, IDT), annealed, and cloned into the BbsI sites of pDCC6. pDCC6 was a gift from Peter Duchek (Addgene plasmid \# 59985).

To generate the C-terminal V5 tagged transgene vectors of Chchd2-PA, Chchd2-PB, or P32, cDNA fragments were amplified from the clones (RE73921 and LD29590) by using PCR and inserted the fragments into the pUASTattB plasmid. The point mutations of Chchd2, Chchd2 ${ }^{K 164 Q}$, Chchd2 $2^{G 88 V}$, and $C h c h d 2^{S 81 L}$ were generated by site directed mutagenesis, the mutations were made on the Chchd2$P A$ cDNA and inserted into pUASTattB vector containing $\mathrm{C}$-terminal V5 tag. To generate constructs expressing Cterminal HA or Flag tagged Opal, full length Opal from the cDNA clone (GH13793) were subcloned into pUASTattB-3 $\times$ HA and pUASTattB-flag vectors, respectively. To generate pUASTattB-YME1L-3 $\times$ HA, YME1L cDNA from clone (GH14313) was amplified and inserted into the pUASTattB- $3 \times$ HA vector.

For the constructs used for mammalian studies: To obtain expression vectors for $\mathrm{C}$-terminal Myc tagged $\mathrm{CHCHD}$, full length human $C H C H D 2$ (IOH3869) was subcloned into pcDNA3.1-3 $\times$ HA. Human CIQBP cDNA (IOH3448) was inserted into the pcDNA3.1 vector containing V5 or HA tag.

The transgenic flies were generated by a standard procedure. The RNAi stocks were obtained from Tsinghua Fly center and Vienna Drosophila RNAi Center. the Gal4 stocks were obtained from the Bloomington Stock Center. The genotypes of the fly strains generated in the paper are described in supplemental Tables.

\section{CRISPR-Cas9 mediated knock-out and knock-in}

Chchd2 knockout flies were produced by using CRISPR/ Cas9 technique as described before [40]. The Chchd2-gRNA (GTGTCCAATGGCTGAGCCCA) was design to target the Chchd 2 coding region. The $g R N A$ along with in vitro transcribed Cas 9 mRNA were injected into w1118 embryos. The F0 and F1 heterozygous mutants were selected by sequencing.

To generate the Opa1-(HA) knock-in fly, pDCC6-Opa1stop gRNA plasmid was mixed with pBluescript SK 
Fig. 6 The interaction between Chchd 2 and P32 modulates mitochondrial fission through regulating the levels of Opa1. a In the wild type animals, P32 binds to Chchd2 and YME1L.

The interaction between P32 and YME1L facilitate the degradation of Opa1. Chchd2 competes with YME1L for P32 and therefore maintains the proper levels of Opa1. b In the Chchd 2 mutants, extra amount of P32 is available to YME1L, which facilitates the degradation of Opa1 and therefore leads to mitochondrial fragmentation.

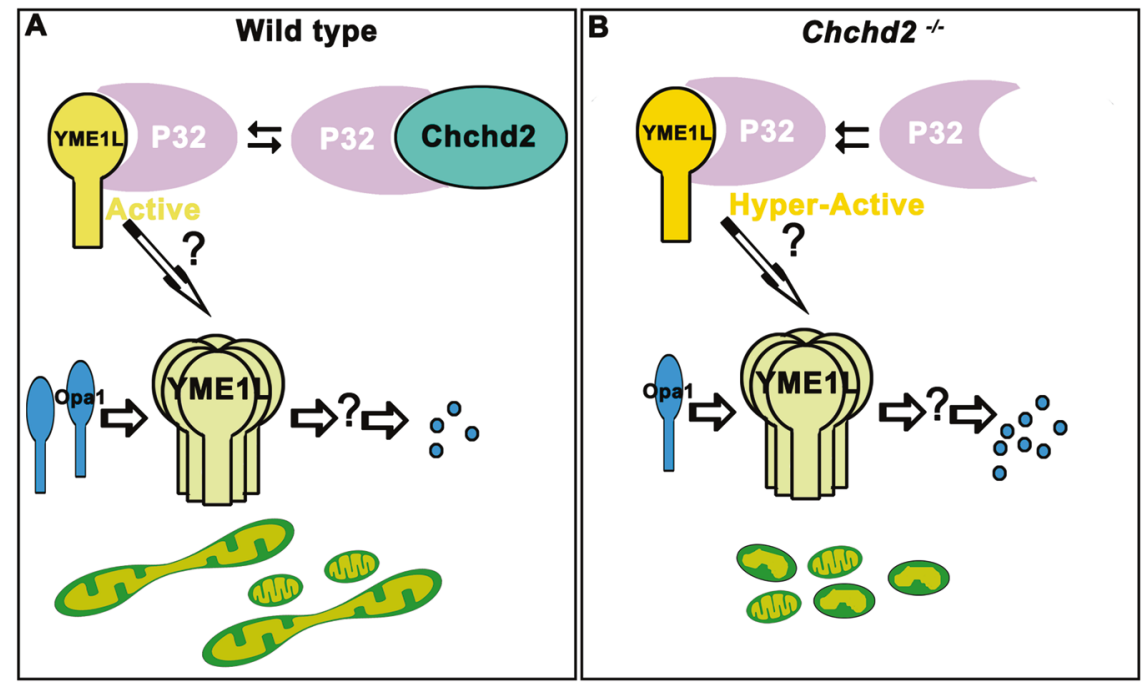

(+)-Opa1-3xHA-stop HR donor vector for w1118 strain injection. All embryo injection and mutant lines selection procedures had been processed by WellGenetics Inc.

\section{Fly strains}

The fly stocks information and the genotypes of the fly stains used in this study were listed in the supplementary data.

\section{Cell culture and transfection}

S2 cells were cultured in a $25^{\circ} \mathrm{C}$ incubator in Schneider insect cell culture medium (Sigma-Aldrich, S0146). Hela cells were cultured in a $37{ }^{\circ} \mathrm{C}$ incubator with $5 \% \mathrm{CO}_{2}$ in Dulbecco's modified Eagle's medium (Life Technologies, C11995500CP). Both the culture mediums were supplemented with $10 \%$ fetal bovine serum (Life Technologies, 10091148) and $50 \mathrm{IU} / \mathrm{mL}$ penicillin/streptomycin (Biological Industries, 03-031-1B). For the transfection, plasmids were transfected into cells by using transfectamine 2000 (Life Technologies, 52887). Forty eight hours after transfection, the cells were harvested and used for immunoprecipitation assay or western blotting.

\section{Immunohistochemistry}

For fly tissues, fat bodies from 3rd instar larvae were fixed in PBS $\left(137 \mathrm{mM} \mathrm{NaCl}, 2.7 \mathrm{mM} \mathrm{KCl}, 10 \mathrm{mM} \mathrm{Na} 2 \mathrm{HPO}_{4}, 2\right.$ $\mathrm{mM} \mathrm{KH}_{2} \mathrm{PO}_{4}$, pH 7.4) with $4 \%$ paraformaldehyde (SigmaAldrich, 158127) for $30 \mathrm{~min}$, followed by washing with PBS for 3 times. Then, the samples were mounted in $80 \%$ glycerol (Sangon Biotech, A100854) with $5 \mathrm{ng} / \mu \mathrm{L}$ DAPI (Sigma-Aldrich, D9542) followed by confocal microscopy (Cal Zeiss, LSM710, Oberkochen, Germany). For the brain tissues from adult flies, the brains were dissected in cold PBS with $4 \%$ paraformaldehyde and fixed for another $4 \mathrm{~h}$, followed by washing with PBST (PBS $+0.5 \%$ Triton X100 (Sangon Biotech, T0694)) 3 times. The brains were blocked with 5\% Bovine Serum Albumin (Sangon Biotech, A600903) for $2 \mathrm{~h}$. The brains were incubated with anti-TH antibody for $48 \mathrm{~h}$ in $4{ }^{\circ} \mathrm{C}$ followed by washing with PBST 3 times and incubated with secondary antibody at room temperature for $2 \mathrm{~h}$. After washed 3 times with PBST, the brains were mounted in $80 \%$ glycerol followed by confocal microscopy. For S2 cells and Hela cells, $24 \mathrm{~h}$ after transfection, cells were fixed with $4 \%$ paraformaldehyde for $30 \mathrm{~min}$, permeabilized with $0.1 \%$ Triton X-100 for $10 \mathrm{~min}$, and blocked with 5\% Bovine Serum Albumin for $30 \mathrm{~min}$. The cells were then immunestained with primary antibodies against indicted antibodies overnight in $4{ }^{\circ} \mathrm{C}$, and followed by washing and fluorescent dye-conjugated secondary antibodies staining for $1 \mathrm{~h}$ at RT. Then the cells were mounted in $80 \%$ glycerol with $5 \mathrm{ng} / \mu \mathrm{L}$ DAPI followed by confocal microscopy.

\section{Climbing assays}

Groups of 10 males in the same age were transferred into the climbing apparatus and incubated for $5 \mathrm{~min}$ at room temperature for the environmental acclimatization. Gently tap the flies down to the bottom of the vial and measure the number of flies that can climb above the 8 -cm mark by $10 \mathrm{~s}$ after the tap. Ten trials were performed for each group, allowing for 1-min rest period between each trial. Six different groups were analyzed for each genotype. Males (2-day-old and 30-day-old) were measured. The average pass rate was calculated for flies of all ages. 


\section{Transmission electron microscopy}

For the fly heads, eyes, and thorax muscle system, those samples were dissected and fixed at $4{ }^{\circ} \mathrm{C}$ in $2 \%$ paraformaldehyde (Electron Microscopy Sciences, 15710); 2\% glutaraldehyde (Electron Microscopy Sciences, 16020); $0.1 \mathrm{M}$ sodium cacodylate ( $\mathrm{pH}$ 7.2) (Electron Microscopy Sciences, 12201). Then, they were post-fixed in $2 \% \mathrm{OsO}_{4}$ (Electron Microscopy Sciences, 19152). The eyes and thorax muscle samples were cut into 50-nm thin sections and stained with 4\% uranyl acetate (Electron Microscopy Sciences, 22400) and 2.5\% lead nitrate (Electron Microscopy Sciences, 17800) for electron microscopy analysis (Hitachi Ltd., HT7700, Tokyo, Japan). For the semi-thin sections of brain tissues, the tissues were cut into $1.5-\mu \mathrm{m}$ thin sections and stained with toluidine blue (Electron Microscopy Sciences, 22050) and examine under a light microscopy (Nikon Corporation, ECLIPSE 80i, Tokyo, Japan).

\section{Real-time RT-PCR}

Total RNA was extracted from S2 cells by using Trizol reagent (Invitrogen, 15596018) according to the manufacturer's protocol. Real-time RT-PCR analysis was performed with a Power SYBR Green PCR Master Mix (Applied Biosystems, 4367659) and an Applied Biosystems 7500 Real-Time PCR System. Relative mRNA levels were calculated by normalizing against the endogenous Act57B mRNA levels (internal control). For each experiment, qPCR reactions were performed in triplicate. The following primers sequences were used: Chchd2 forward primer: 5'GGTCATGGATTGACCTCGCT-3'; Chchd2 reverse primer: 5'-AGAGGGTAAGATCCGCCTGA-3'; Marf forward primer: 5'-AGCCCGAGTGCCAGGAAT-3'; Marf reverse primer: 5'-CCCTGGCGGAAACGAAGAAT-3'; Drp1 forward primer: 5'-GAGTGGAACGCGACCAAAG-3'; Drp1 reverse primer: 5'-AGGATCGTCCCACAACGCT-3'; Opa1 forward primer: 5'- TCCCCAGATTGCGCGAG-3'; Opa1 reverse primer: 5'-CAGCGGGCAGATAGAT.

GCTT-3'; Act57B forward primer: 5'-CATCCTGGC CTCTCTGTCCA-3'; Act57B reverse primer: 5'-GACCA GATGAGCAGGAGACA-3'.

\section{Western blotting}

Fat boy tissues of 3rd instar larvae and thorax muscle from adult flies were dissected in PBS, followed by centrifuging at $5000 \times g$ for $5 \mathrm{~min}$ at $4{ }^{\circ} \mathrm{C}$. Then PBS was removed and $2 \times$ SDS-loading buffer was added. Proteins were separated by SDS-PAGE, and transferred onto a PVDF membrane. The membrane was blocked with 5\% non-fat milk in TBST buffer and incubated with primary antibodies in 5\% non-fat milk (Sangon Biotech, A600669) in TBST overnight at $4{ }^{\circ} \mathrm{C}$. The membranes were then washed and incubated in HRP labeled secondary antibodies (1:5000 in 5\% non-fat milk/TBST) for $1 \mathrm{~h}$ at RT. The membranes were then washed in TBST and incubated with ECL reagents (Cyanagen Srl, XLS3-0020) and exposed. Quantification of protein bands was done with Image $\mathbf{J}$ software. For the culture cells, the cells were collected and washed with PBS for 3 times, followed by adding $1 \times$ SDS-loading buffer to lysate samples.

\section{Immunoprecipitation}

The IP experiments were performed as described before [41].

\section{LC-MS/MS analysis}

The Hela cells were collected and washed with PBS, then the cells were homogenized in Lysis Buffer $(20 \mathrm{mM}$ Tris$\mathrm{HCl}$ pH8.0, $80 \mathrm{mM} \mathrm{NaCl}, 20 \mathrm{mM} \mathrm{NaF}, 5 \mathrm{mM} \mathrm{MgCl}_{2}, 10 \%$ glycerol, $0.1 \%$ Triton-100 and complete protease inhibitors) for $2 \mathrm{~h}$. The lysates then were cleared by centrifuge at $2000 \times g$ for $5 \mathrm{~min}$. Twenty microliter of the CHCHD2 antibody was added to $400 \mu \mathrm{L}$ protein A beads and incubated overnight at $4{ }^{\circ} \mathrm{C}$. Four hundred microliter antiCHCHD2 coated beads slurry was added to the lysate. After incubation for $4 \mathrm{~h}$ at $4{ }^{\circ} \mathrm{C}$, the beads were pelleted for $5 \mathrm{~min}$ and washed 4 times with Lysis Buffer, followed by elution of bound protein with $2 \times$ SDS-loading buffer. Proteins were separated by SDS-PAGE, after migrating about $1-\mathrm{cm}$, the gel were cut and subjected to in-gel digestion for LC-MS/ MS analysis. The LC-MS/MS analysis was carried by National Center for Protein Science, Beijing (Thermo Fisher Scientific, Waltham, Massachusetts, USA). Standard procedures were followed.

\section{Statistical analysis}

The length of mitochondrial length and size of mitochondrial aggregates were quantified from confocal images using Image-Pro Plus 6.0 software. For details, the image was imported into the Image-Pro Plus 6.0 software. Using regular AOI to trace the shape of a tested cell. The fine details of the mitochondrial network were increased by setting the "Sharpen" under the filter of "Enhancement" with the options adjust to " $5 \times 5$ " and "passes" adjust to 2 . The mitochondrial networks were then adjusted to 1 pixel by setting the options of "Thinning" to $22 \%$ under the filter of "Morphological". Then, with the " $2 \times 2$ Square" options of "Deliate" to enlarge the edges of mitochondrial networks to 2 pixels and measure the area of labeled mitochondria. Then final length of the mitochondrial was determined by 
dividing the area by width of 2 . All the images were measured 3 times under the same conditions. The average mitochondrial length was calculated. Then, the relative mitochondrial length was normalized by dividing the average length of mitochondria in the experimental group to that of the control group. The results were analyzed by twotailed unpaired Student's $t$ test or one-way ANOVA with Bonferroni post-hoc test. The data presented as mean values + SEM. A $p$-value $<0.05$ was considered statistically significant.

\section{Antibodies and reagents}

Antibody dilution used: rabbit anti-CHCHD2 (SigmaAldrich, A76006) (IF 1:500); mouse anti-TH (ImmunoStar, Inc., BML-PW8810) (IF: 1:2000); mouse anti-Flag (SigmaAldrich, F3165) (IF 1:1000); mouse anti-V5 (Invitrogen, R96025) (IF 1:400, WB 1:5000); mouse anti-HA.11 (16B12) (Covance Research Products, MMS-101P) (IF 1:500, WB 1:1000); mouse anti-Tubulin (Beyotime Biotechnology, AT819) (WB 1:1000). For the secondary antibody, Alexa Flour 488-conjugated anti-mouse IgG (Invitrogen, A-21202), Alexa Flour 594-conjugated anti-rabbit IgG (Invitrogen, A-21207).

Acknowledgements We are grateful to THFC, BDSC, and DGRC for providing fly strains and cDNA clones and the imaging core facilities in LSI for the technical support. Dr. CT is supported by National Natural Science Foundation of China (31622034, 31571383, 91754103), National Key Research and Developmental Program of China (2017YFC100 1500, 2017YFC1001100), Natural Science Foundation of Zhejiang Province, China (LR16C070001), the National Basic Research Program of China (2014CB943100) and Fundamental research funds for the central universities. Dr. LW is supported by Natural Science Foundation of Zhejiang Province, China (LY19H040014). Dr. CT is a Qianjiang Scholar.

\section{Compliance with ethical standards}

Conflict of interest The authors declare that they have no conflict of interest.

Publisher's note Springer Nature remains neutral with regard to jurisdictional claims in published maps and institutional affiliations.

\section{References}

1. Nunnari J, Suomalainen A. Mitochondria: in sickness and in health. Cell. 2012;148:1145-59.

2. Schrepfer E, Scorrano L. Mitofusins, from mitochondria to metabolism. Mol cell. 2016;61:683-94.

3. Mishra P, Chan DC. Mitochondrial dynamics and inheritance during cell division, development and disease. Nat Rev Mol Cell Biol. 2014;15:634-46.

4. Mishra P, Chan DC. Metabolic regulation of mitochondrial dynamics. J Cell Biol. 2016;212:379-87.

5. Wai T, Langer T. Mitochondrial dynamics and metabolic regulation. Trends Endocrinol Metab: TEM. 2016;27:105-17.
6. Modjtahedi N, Tokatlidis K, Dessen P, Kroemer G. Mitochondrial proteins containing Coiled-Coil-Helix-Coiled-Coil-Helix $(\mathrm{CHCH})$ domains in health and disease. Trends Biochem Sci. 2016;41: 245-60.

7. Imai Y, Meng H, Shiba-Fukushima K, Hattori N. Twin $\mathrm{CHCH}$ Proteins, CHCHD2, and CHCHD10: Key Molecules of Parkinson's Disease, Amyotrophic Lateral Sclerosis, and Frontotemporal Dementia. Int J Mol Sci. 2019,20:908.

8. Bannwarth S, Ait-El-Mkadem S, Chaussenot A, Genin EC, LacasGervais S, Fragaki K, et al. A mitochondrial origin for frontotemporal dementia and amyotrophic lateral sclerosis through CHCHD10 involvement. Brain: a J Neurol. 2014;137(Pt 8): 2329-45.

9. Penttila S, Jokela M, Bouquin H, Saukkonen AM, Toivanen J, Udd B. Late onset spinal motor neuronopathy is caused by mutation in CHCHD10. Ann Neurol. 2015;77:163-72.

10. Auranen M, Ylikallio E, Shcherbii M, Paetau A, Kiuru-Enari S, Toppila JP, et al. CHCHD10 variant p.(Gly66Val) causes axonal Charcot-Marie-Tooth disease. Neurol Genet. 2015;1:e1.

11. Genin EC, Plutino M, Bannwarth S, Villa E, Cisneros-Barroso E, Roy M, et al. CHCHD10 mutations promote loss of mitochondrial cristae junctions with impaired mitochondrial genome maintenance and inhibition of apoptosis. EMBO Mol Med. 2016;8:58-72.

12. Genin EC, Bannwarth S, Lespinasse F, Ortega-Vila B, Fragaki K, Itoh $\mathrm{K}$, et al. Loss of MICOS complex integrity and mitochondrial damage, but not TDP-43 mitochondrial localisation, are likely associated with severity of CHCHD10-related diseases. Neurobiol Dis. 2018;119:159-71.

13. Zhou W, Ma D, Sun AX, Tran HD, Ma DL, Singh BK, et al. PDlinked CHCHD2 mutations impair CHCHD10 and MICOS complex leading to mitochondria dysfunction. Hum Mol Genet. 2019;28:1100-16.

14. Funayama M, Ohe K, Amo T, Furuya N, Yamaguchi J, Saiki S, et al. CHCHD2 mutations in autosomal dominant late-onset Parkinson's disease: a genome-wide linkage and sequencing study. Lancet Neurol. 2015;14:274-82.

15. Aras S, Pak O, Sommer N, Finley R Jr., Huttemann M, Weissmann $\mathrm{N}$, et al. Oxygen-dependent expression of cytochrome $\mathrm{c}$ oxidase subunit 4-2 gene expression is mediated by transcription factors RBPJ, CXXC5 and CHCHD2. Nucleic Acids Res. 2013;41:2255-66.

16. Aras S, Bai M, Lee I, Springett R, Huttemann M, Grossman LI. MNRR1 (formerly CHCHD2) is a bi-organellar regulator of mitochondrial metabolism. Mitochondrion. 2015;20:43-51.

17. Baughman JM, Nilsson R, Gohil VM, Arlow DH, Gauhar Z, Mootha VK. A computational screen for regulators of oxidative phosphorylation implicates SLIRP in mitochondrial RNA homeostasis. PLoS Genet. 2009;5:e1000590.

18. Liu Y, Clegg HV, Leslie PL, Di J, Tollini LA, He Y, et al. CHCHD2 inhibits apoptosis by interacting with Bcl-x L to regulate Bax activation. Cell Death Differ. 2015;22:1035-46.

19. Meng H, Yamashita C, Shiba-Fukushima K, Inoshita T, Funayama M, Sato S, et al. Loss of Parkinson's disease-associated protein CHCHD2 affects mitochondrial crista structure and destabilizes cytochrome c. Nat Commun. 2017;8:15500.

20. Mao Z, Davis RL. Eight different types of dopaminergic neurons innervate the Drosophila mushroom body neuropil: anatomical and physiological heterogeneity. Front Neural Circuits. 2009;3:5.

21. Hewitt VL, Whitworth AJ. Mechanisms of Parkinson's Disease: Lessons from Drosophila. Curr Top Dev Biol. 2017;121: 173-200.

22. Sandoval H, Yao CK, Chen K, Jaiswal M, Donti T, Lin YQ, et al. Mitochondrial fusion but not fission regulates larval growth and synaptic development through steroid hormone production. Elife 2014,3:e03558. 
23. Verstreken P, Ly CV, Venken KJ, Koh TW, Zhou Y, Bellen HJ. Synaptic mitochondria are critical for mobilization of reserve pool vesicles at Drosophila neuromuscular junctions. Neuron. 2005;47: $365-78$.

24. Deng H, Dodson MW, Huang H, Guo M. The Parkinson's disease genes pink1 and parkin promote mitochondrial fission and/or inhibit fusion in Drosophila. PNAS. 2008;105:14503-8.

25. Yang Y, Ouyang Y, Yang L, Beal MF, McQuibban A, Vogel H, et al. Pink1 regulates mitochondrial dynamics through interaction with the fission/fusion machinery. PNAS. 2008;105:7070-5.

26. Song Z, Chen H, Fiket M, Alexander C, Chan DC. OPA1 processing controls mitochondrial fusion and is regulated by mRNA splicing, membrane potential, and Yme1L. J Cell Biol. 2007;178: 749-55.

27. Head B, Griparic L, Amiri M, Gandre-Babbe S, van der Bliek AM. Inducible proteolytic inactivation of OPA1 mediated by the OMA1 protease in mammalian cells. J Cell Biol. 2009;187: 959-66.

28. Anand R, Wai T, Baker MJ, Kladt N, Schauss AC, Rugarli E, et al. The i-AAA protease YME1L and OMA1 cleave OPA1 to balance mitochondrial fusion and fission. J Cell Biol. 2014;204: 919-29.

29. Wei Y, Vellanki RN, Coyaud E, Ignatchenko V, Li L, Krieger JR, et al. CHCHD2 Is Coamplified with EGFR in NSCLC and Regulates Mitochondrial Function and Cell Migration. Mol Cancer Res 2015;13:1119-29.

30. Burstein SR, Valsecchi F, Kawamata H, Bourens M, Zeng R, Zuberi $\mathrm{A}$, et al. In vitro and in vivo studies of the ALS-FTLD protein CHCHD10 reveal novel mitochondrial topology and protein interactions. Hum Mol Genet. 2018;27:160-77.

31. Lutas A, Wahlmark CJ, Acharjee S, Kawasaki F. Genetic analysis in Drosophila reveals a role for the mitochondrial protein p32 in synaptic transmission. G3. 2012;2:59-69.
32. Purandare N, Somayajulu M, Huttemann M, Grossman LI, Aras S. The cellular stress proteins CHCHD10 and MNRR1 (CHCHD2): Partners in mitochondrial and nuclear function and dysfunction. J Biol Chem. 2018;293:6517-29.

33. MacVicar T, Langer T. OPA1 processing in cell death and disease - the long and short of it. J Cell Sci. 2016;129:2297-306.

34. Ishihara N, Fujita Y, Oka T, Mihara K. Regulation of mitochondrial morphology through proteolytic cleavage of OPA1. EMBO J. 2006;25:2966-77.

35. Duvezin-Caubet S, Koppen M, Wagener J, Zick M, Israel L, Bernacchia A, et al. OPA1 processing reconstituted in yeast depends on the subunit composition of the m-AAA protease in mitochondria. Mol Biol Cell. 2007;18:3582-90.

36. McQuibban GA, Lee JR, Zheng L, Juusola M, Freeman M. Normal mitochondrial dynamics requires rhomboid-7 and affects Drosophila lifespan and neuronal function. Curr Biol. 2006;16:982-9.

37. Whitworth AJ, Lee JR, Ho VM, Flick R, Chowdhury R, McQuibban GA. Rhomboid-7 and HtrA2/Omi act in a common pathway with the Parkinson's disease factors Pink1 and Parkin. Dis Models Mechanism. 2008;1:168-74. discussion 173

38. Storz P, Hausser A, Link G, Dedio J, Ghebrehiwet B, Pfizenmaier $\mathrm{K}$, et al. Protein kinase $\mathrm{C}$ [micro] is regulated by the multifunctional chaperon protein p32. J Biol Chem. 2000;275:24601-7.

39. Yagi M, Uchiumi T, Takazaki S, Okuno B, Nomura M, Yoshida $\mathrm{S}$, et al. p32/gC1qR is indispensable for fetal development and mitochondrial translation: importance of its RNA-binding ability. Nucleic Acids Res. 2012;40:9717-37.

40. Yu Z, Ren M, Wang Z, Zhang B, Rong YS, Jiao R, et al. Highly Efficient Genome Modifications Mediated by CRISPR/Cas9 in Drosophila. Genetics. 2013;195:289-91.

41. Tong C, Jiang J. Using immunoprecipitation to study proteinprotein interactions in the Hedgehog-signaling pathway. Methods Mol Biol. 2007;397:215-29. 